\title{
Dormancy in Cryptococcus neoformans: 60 years of accumulating evidence
}

\author{
Alexandre Alanio $0^{1,2,3}$ \\ 'Laboratoire de Parasitologie-Mycologie, Groupe Hospitalier Saint-Louis-Lariboisière-Fernand-Widal, Assistance Publique-Hôpitaux de Paris (AP-HP), Paris, France. ${ }^{2}$ Molecular Mycology Unit, \\ CNRS UMR 2000, National Reference Center for Invasive Mycoses and Antifungals (NRCMA), Institut Pasteur, Paris, France. ${ }^{3}$ Université de Paris, Paris, France.
}

\begin{abstract}
Cryptococcus neoformans is an opportunistic yeast that is present worldwide and interacts with various organisms. In humans, it is responsible for cryptococcosis, a deadly invasive fungal infection that represents around 220,000 cases per year worldwide. Starting from the natural history of the disease in humans, there is accumulating evidence on the capacity of this organism to enter dormancy. In response to the harsh host environment, the yeast is able to adapt dramatically and escape the vigilance of the host's immune cells to survive. Indeed, the yeast exposed to the host takes on pleiotropic phenotypes, enabling the generation of populations in heterogeneous states, including dormancy, to eventually survive at low metabolic cost and revive in favorable conditions. The concept of dormancy has been validated in $C$. neoformans from both epidemiological and genotyping data, and more recently from the biological point of view with the characterization of dormancy through the description of viable but nonculturable cells.
\end{abstract}

Cryptococcus neoformans is a basidiomycetous opportunistic yeast that is widely present in the environment. It causes human cryptococcosis, which mainly affects immunocompromised patients and presents as a meningoencephalitis (1) that is lethal without treatment. Clinical presentation is often diagnosed late because clinical symptoms are initially mild with a subacute to chronic evolution (2).

Humans are exposed to C. neoformans from the environment. In nature, this fungus can survive the predation of various organisms ranging from protozoans to metazoans through ready-made virulence traits (3). C. neoformans interacts closely with unicellular or multicellular organisms (2-4) and with cells dedicated to innate immune responses in metazoans (macrophages, dendritic cells, natural killer lymphocytes) with various propensity to be phagocytosed and killed (4-6). C. neoformans is a facultative intracellular pathogen (7). Interaction of C. neoformans with host cells can lead to phagocytosis, yeast replication within the phagolysosome, and is sometimes associated with host cell lysis or with nonlytic exocytosis or cell-to-cell transfer and eventually killing of the yeast $(8-13)$. These phases have been well studied in different models of interaction with host cells but mainly within macrophages. Indeed, intracellular persistence and multiplication in immune cells provide advantages to the fungus by allowing escape from the immune response and later dissemination through epithelial barriers $(14,15)$.

Characteristics of the infection depend on both hosts and microbial factors. Fungal factors described as virulence factors influence the outcome of infection, according to data obtained in the mouse model of cryptococcosis (16), but also in vitro (17) and

Conflict of interest: AA received a grant $(€ 102,000)$ from the French National Research Agency in 2019 as a partner in the French-German call to fight against antimicrobial resistance.

Copyright: (c) 2020, American Society for Clinical Investigation.

Reference information: J Clin Invest. 2020;130(7):3353-3360.

https://doi.org/10.1172/JCl136223. in humans $(8,18)$. Microbial adaptation to the hosts is complex and has been studied globally in lungs using histopathology (7) and global transcriptome analysis upon ameba (19) and macrophage ingestion (20) or upon early infection of mice and rabbits $(21,22)$. Quiescence or dormancy is one such adaptation that appears successful for enhancing the fungus's ability to survive, persist, reactivate, and then disseminate (23).

About 60 years of research focusing on how C. neoformans causes infection in humans is available in the literature, leading to the recent biological demonstration of dormancy in this organism. This Review aims to summarize this 60 years of research, starting from the knowledge of human infection and ending with the characterization of dormancy biologically. The Review is assembled to elucidate how this knowledge has been integrated to lead to more recent findings on the biology of $C$. neoformans characterizing dormancy, focusing on (a) the description of the natural history of C. neoformans infection in humans; (b) the concept of dormancy in fungi; and dormancy in C. neoformans (c) in vivo and (d) in vitro. To finish, a section is dedicated to (e) the relevance of the biological findings to human infection and a discussion of unsolved questions that can provide the bases of future work in the field.

\section{Natural history of cryptococcosis in humans}

Cryptococcosis is one of the most frequent invasive fungal infections in humans worldwide (24). The vast majority of patients with cryptococcosis are $\mathrm{HIV}^{+}$, mostly those with fewer than $100 \mathrm{CD}^{+} \mathrm{T}$ cells per microliter. Nevertheless, in Western countries, the number of cryptococcosis cases recorded in $\mathrm{HIV}^{-}$individuals becomes higher than the number in $\mathrm{HIV}^{+}$patients (25). Immunocompromised $\mathrm{HIV}^{-}$patients at risk of cryptococcosis are mainly solid organ transplant recipients, patients with systemic autoimmune disease, and those with hematological malignancies (26).

The natural history of cryptococcosis is described as following two main routes. The first, although rare, occurs after exposure 
to C. neoformans while immunocompromised, leading to rapidly progressive cryptococcosis; the second is reminiscent of tuberculosis, with a phase of latency followed by reactivation and dissemination. This second route appears to be the main mechanism of infection and so will be further developed in this Review.

\section{First route of infection: ready-made for disease}

Confronted with the need to survive in nature and to survive different hosts in different environments, C. neoformans has selected ready-made virulence traits (3). From a deterministic point of view, the C. neoformans population also needs diversity to survive predators harboring different killing propensities. The plasticity of the C. neoformans genome could lead to this diversity (27). C. neoformans and C. gattii are haploid organisms that can be found as diploid organisms both in nature and in hosts $(28,29)$. Generation of hybrids is possible between the varieties grubii (serotype A) and neoformans (serotype D) $(29,30)$ but also between C. neoformans and C. gattii, again illustrating this plasticity (31).

C. neoformans has long been associated with pigeon droppings (32). Indeed, pigeon fanciers are known to have higher levels of anti-C. neoformans antibodies than control individuals (33). The presence of C. neoformans in human dwellings was a risk factor (odds ratio 2.05) for the development of cryptococcosis in $\mathrm{HIV}^{+}$ patients from Brazil (34). C. gattii has also been found in indoor environments in Brazil, although links with human cryptococcosis have not been demonstrated (35). Several cases of cryptococcosis have been reported in immunosuppressed patients in contact with birds (pigeons, parrots, cockatoos, cockatiels) (36-39). The presence of $C$. neoformans has also been observed in the excreta of some zoo animals (tawny frogmouths, palm cockatoos, military macaws, gray parrots) (40). Nosocomial cases of cryptococcosis acquired in various hospital settings have also been suspected (41, 42). Transmission of $C$. neoformans through transplanted deep organs from a contaminated donor has occurred $(43,44)$, with, in some cases, the demonstration of the same strain in different patients with transplanted organs from the same donor (45).

Primary cryptococcosis initiates with lung involvement and then disseminates from the lung in immunocompromised hosts. Primary pulmonary cryptococcosis is observed in immunocompetent and immunocompromised hosts. It can be recognized within a broad range of presentation, from isolated asymptomatic nodules that can mimic cancer lesions to more disseminated lesions of the lung with respiratory failure (46-48). Primary cutaneous cryptococcosis is also a clinical entity that happens after environmental inoculation in immunocompetent or immunocompromised hosts $(49,50)$.

\section{Second route of infection: ready-made for latency}

The majority of cases of cryptococcosis arise from a natural history of infection following three steps: primary infection in childhood and immune control, followed by a silent phase of latency that can last for years, and finally reactivation and dissemination that are responsible for the symptoms of the disease mainly occurring upon immunosuppression.

Early environmental exposure. Inhalation of aerosolized particles from soil (desiccated yeasts or basidiospores) is thought to be the major route of infection in humans (51). Primary infection with C. neoformans occurs mainly in immunocompetent children as demonstrated by serologic studies with unrecognized (asymptomatic) infection as the main clinical presentation. The proportion of children immunized against $C$. neoformans increases with age. Acquisition of cryptococcal antibodies begins very early (1 year) with minimal reactivity of the sera. After 5 years, $70 \%$ of children react with $C$. neoformans antigens (52). However, the acquisition of anticryptococcal humoral immunity varies among geographic areas. Cryptococcal antibodies are very common in Bronx children but not in another New York area (Dutchess County), nor are they common in Manila, the Philippines, another densely populated urban area (53). Environmental exposure may depend on climatic and environmental factors (temperature, humidity, pigeon density), but also on human sociological factors (habitat conditions, financial resources). These findings support epidemiological data revealing that cryptococcosis in immunocompromised individuals is more prevalent in some areas of the world, especially in Africa $(24,54)$.

Notably, C. gattii exposure and primoinfection does not follow the same epidemiological trends as C. neoformans, based on studies realized in endemic areas in animals and humans $(55,56)$.

Latency. Serologic evidence of early cryptococcal immunity in immunocompetent hosts without recognized infection seems paradoxical considering the very low frequency of cryptococcosis in immunocompetent hosts. However, immune control of yeasts by immunocompetent hosts following primoinfection is possible, with latency of the disease or complete clearance of the fungus as a consequence. Immunocompetent adults frequently exposed to $C$. neoformans had positive skin test but did not develop clinical disease (57). Autopsy studies have raised the hypothesis that pulmonary granulomas could be the site of persistence, because C. neoformans is observed in subpleural nodules and draining lymph nodes in immunocompetent and immunocompromised hosts (58). Indeed, there have been several reports of $C$. neoformans lymphadenitis being exclusively found in and isolated from lymph nodes, arguing that initial immune control of the yeast operates in lymph nodes (59-65). From recent and old reports, the lymph nodes associated with lymphadenitis correspond to granuloma composed of epithelioid cells, giant cells, and necrosis surrounded by a T cell infiltrate together with yeasts $(64,66,67)$.

Analysis of clinical isolates of C. neoformans var. grubii recovered in France from patients born in Africa (who moved to France with a median of 110 months elapsing before isolation of the yeast in France) revealed that yeast genotypes from these patients clustered together, distinct from the yeast genotypes recovered from patients born in Europe (68). This study is the main epidemiological evidence for this latency stage of the disease. This latency can be translated into the capacity of dormancy of the yeasts, which appears to be the more plausible explanation from the point of view of the biology of the organism. The same conclusion is also drawn from a serologic survey of solid organ transplant recipients (immunocompromised hosts). Interestingly, sera obtained before and after transplantation from transplanted patients with cryptococcosis were compared with sera from control transplanted patients without history of cryptococcosis. Among patients with cryptococcosis, half exhibited antibody reactivity against $C$. neoformans only after transplantation, suggesting that they were exposed and developed the disease after transplantation during immunosuppression. 
But for the other half of the patients, antibody reactivity against C. neoformans was found before transplantation; these patients' early development of cryptococcosis after transplantation suggests that reactivation and dissemination occur rapidly after transplantation from a preexisting isolate in transplant recipients, thus validating again the latency phenomenon (69). Additionally, a report of C. gattii infections in patients who travelled to endemic areas years or months before the Vancouver Island C. gattii outbreak provides more evidence for latency (70).

Reactivation. The earliest manifestation of reactivation is observed in individuals in whom asymptomatic cryptococcal antigenemia is detected (71-73). Viable yeasts are not recovered from clinical sample at this step, but treatment is mandatory to prevent symptoms and dissemination $(74,75)$. Pulmonary cryptococcosis is a well-described clinical entity that can evolve differentially depending on the immune status of the hosts. In immunocompetent hosts, C. neoformans does not usually disseminate, whereas the possibility of dissemination in immunocompromised patients is high. It is likely that dissemination occurs after reactivation of lung-persistent yeasts, crossing the lung epithelial barrier and disseminating through capillary blood $(76,77)$. However, abnormal chest x-ray or CT scan was observed in $39 \%$ of $\mathrm{HIV}^{+}$patients and $55 \%$ of $\mathrm{HIV}^{-}$patients at diagnosis, although dissemination represented $60.6 \%$ and $38.5 \%$ of the cases, respectively (1). However, pulmonary symptoms are not the main clinical manifestation of cryptococcosis in immunocompromised patients. Indeed, most are diagnosed at the stage of dissemination or meningoencephalitis (1). Cryptococcosis is characterized by a high frequency of central nervous system involvement with positive cerebrospinal fluid and dissemination through blood. Cryptococcosis is more severe in male $\mathrm{HIV}^{+}$patients and those infected with C. neoformans serotype A (1). Acute cryptococcal meningoencephalitis (CM) is always fatal without antifungal therapy (78). Treatment of CM requires an antifungal therapy induction based on amphotericin $\mathrm{B}$ and flucytosine (79). Based on recent large clinical trials in African settings, 1 week of amphotericin B combined with oral flucytosine followed by high-dose fluconazole is now recognized as the reference therapy $(75,80)$. Mycological failure after 2 weeks of induction is recognized as a factor of bad prognosis, which requires continuation of the induction therapy (79). Mycological failure is independently associated with initial dissemination, high serum antigenemia ( $>1: 512)$, and lack of initial flucytosine treatment $(1$, $80,81)$. The 3 -month mortality rate during the management of acute CM approximates $15 \%$ to $20 \%$ in Western countries despite adequate treatment and management. It is still not clear whether this mortality rate is due to individuals' immune status, genetic factors (82), fungal determinants, or a combination of these. Nevertheless, two reports clearly identified that fungal determinants specific to the strain are responsible for a given phenotype of interaction with host cells (high phagocytosis, high intracellular proliferation) that is associated with mortality in patients $(8,83)$.

\section{The concept of dormancy in fungi}

All microorganisms are exposed to periodic constraint conditions and react by inhibiting their growth, entering into a nonreplicative state called quiescence or dormancy $(84,85)$. Three main strategies can be delineated in these conditions. The first is the "bust and bloom" strategy (85), by which the microorganism population will grow rapidly with growth maximization, but upon nutrient exhaustion, the majority of the individuals will die, with only few cells surviving. These residual cells will resume growth rapidly upon exposure to nutrients (86). The second strategy is quiescence, in which the bulk of the population exposed to a nutrient-limited environment will arrest or slow growth to enter a viable, nonreplicating state for a long time. This can last months or years for Mycobacterium tuberculosis (87). These cells keep a baseline and specific metabolic capacity, maintain their membrane potential, and do not undergo major morphological change (88). The third strategy is called true dormancy, with sporulation as the purest form, in which an asymmetrical replication leads to the formation of a metabolically inactive spore (89). The spore harbors specific morphology but shares some biological features with quiescent cells.

Quiescence in Saccharomyces cerevisiae has been studied for a long time. Recently, a strain of $S$. cerevisiae was found "alive" in bottles of beer and champagne from the 18th century found in a shipwreck in the Baltic Sea, suggesting that this phenomenon can last for years in specific conditions. Quiescent yeasts are mainly obtained from cultures grown to saturation in glucose-rich media (stationary phase) where all nutrients have been consumed. Different phases have been described, including (a) a first phase of glycogen production upon rarefaction of glucose (at about 50\%) (90), followed by (b) the regulation of trehalose before and after glucose exhaustion. Then, (c) the yeasts undergo a phase of diauxic shift (following glucose depletion) in which growth is slow and metabolism is adapted to limitation of nutrients, relying on respiratory growth of nonfermentable sugars such as ethanol or acetate with a switch toward respiration, fatty acid pathway, and glyoxylate cycle pathway and, as a consequence, increased formation of antioxidant defenses (scavenging of ROS) (91). The yeast population obtained in the stationary phase is described as a heterogeneous population including quiescent cells (composed of daughter and young mother cells) but also nonquiescent cells, which lose their ability to accumulate ROS, exhibit genomic instability, and become senescent or apoptotic (92).

In C. neoformans, growth arrest in the $G_{1}$ or $G_{2}$ period has been demonstrated in the stationary phase (93). No specific morphological differences in the mitochondrial apparatus were observed in the logarithmic versus the stationary phase (Figure 1 and ref. 94). No comprehensive analysis of the metabolism of C. neoformans in the stationary compared with the logarithmic phase existed until recently, as part of the investigation of a specific phenotype observed upon exposure to drastic conditions (95).

\section{Dormancy in C. neoformans in vivo}

The body of evidence for dormancy comprises various parameters, primarily viability, which should not be based on culturability, reactivation upon specific stimuli, or specific biological activity. Measurement of viability requires the use and adaptation of tools available to test viability versus death in mammalian cells (23). For a long time, viability and its mirror, killing or death of C. neoformans, were investigated using CFU counting (96). Other means to assess the viability or death of yeast have now been developed, including the use of intercalating dyes such as propidium iodide that are able to diffuse and stain the DNA of the yeast only if the extracellular 


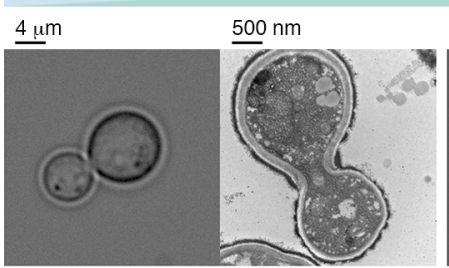

Logarithmic phase Normal growth CFU+

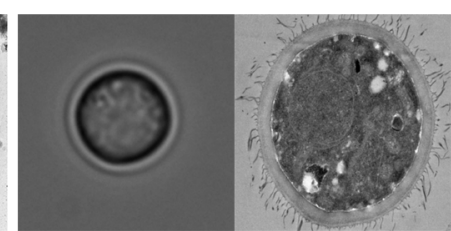

Stationary phase Quiescence CFU+
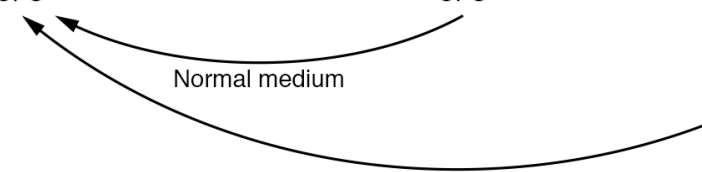

Pantothenic acid (vitamin B5)

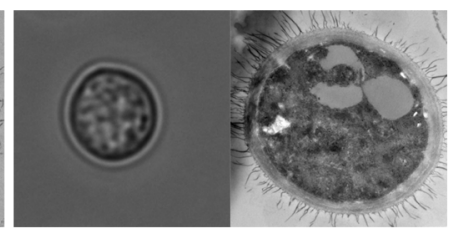

VNBC

Dormancy

CFU-

Figure 1. Schematic representation of the evolution of $C$. neoformans phenotypes and morphology upon incubation under progressive nutrient deprivation and anaerobiosis. The reference strain $\mathrm{H} 99$ was used in all conditions. STAT: stationary phase; yeast peptone dextrose (YPD), 22 hours with agitation at $150 \mathrm{rpm}$. VBNC: after incubation for 8 days in anaerobiosis and nutrient deprivation. DEAD: morphology of dead cells called Drop Cn, including one or two large vacuole-like structures. Each panel shows optical microscopy (left) and electron microscopy (right) images. Yeast cells under agitation and in glucose-rich medium (YPD) are actively multiplying in logarithmic phase (LOC). Quiescent yeasts are culturable (STAT) and do not need specific stimuli to grow in normal glucose-rich medium (YPD). Dormant yeasts (VBNC) are not spontaneously culturable and need a trigger stimulus for reactivation (addition of pantothenic acid). Dead yeasts (DEAD) are irreversibly unable to grow again (refs. 23, 95).

membrane loses integrity (97). Another means is based on Live/ Dead stains that are not based on DNA staining but on the presence of intracellular esterase released if the cytoplasm is no longer intact (95). These allow assessment of viability or death using flow cytometry. Other dyes can be used with the same principle $(23,98)$. These methods assume that a dead yeast cell will lose membrane integrity, which may not be necessary at first. Apoptosis should also be checked in this context to determine whether the cell is oriented toward cell death or will remain viable. The existence of apoptosis in fungi is debated (99), but there is evidence for the presence of caspase-like proteins in C. neoformans (100) that could act as effectors of mechanisms related to caspase-dependent cell death. Nevertheless, apoptosis in fungi cannot be directly equated with what is known in mammalian cells $(99,101)$.

In C. neoformans' stationary phase, it was shown that only a small proportion of the population was unable to grow and was considered dead (23). From in vivo experiments and interaction with macrophages, it has been shown that yeast cells were able to keep their round shape and capsule although dead as shown with different means (23). These dead C. neoformans yeast cells have been called Drop Cn owing to the presence of a large central vesicle inside the cell. The cell wall was shown to be thicker than that in stationary-phase yeasts (Figure 1). In these dead cells, the intracellular content is collapsed around vesicles including remaining membranes (stained with MDY64) and nucleic acids (stained with SYTO85) but with no organized nucleus (negative DAPI staining) and no mitochondria (negative MitoTracker staining) (23). These cells were able to retain CMFDA staining (glutathione staining) in their remaining capsule and cell wall, which was supposed to be intracellular, producing fluorescence artifacts, allowing detection despite being dead. To prevent such bias, multispectral imaging flow cytometry was used, allowing observation of the fluorescence within the cells to assess location (23). Apart from those dead cells, this study highlighted that a heterogeneous population of yeasts was generated in the lungs of infected mice and upon macrophage interaction. Indeed, the view of the existence of a homogeneous population of yeasts in specific conditions turns out to be inadequate, raising the question of the accuracy of studies dealing with analysis of the global population of yeasts recovered in specific settings. Nevertheless, global transcription analyses supported the idea of fungal adaptation to hostile environments such as the macrophage phagolysosome $(19,20,102)$, inside amebae (19), in the lung during murine infection (21), in the central nervous system of rabbits (22), or in human cerebrospinal fluid (103).

Heterogeneous populations generated during murine infection included (a) active yeasts able to bud and multiply, (b) dead yeasts, and also (c) a population of more dormant yeasts. These dormant yeasts were less prone to grow in comparison with the stationary phase, which is already considered as a state in which almost all yeasts are quiescent. This explains why these cells have been called dormant instead of quiescent cells (23). These cells also had a decreased response to stress (low glutathione production), increased mitochondrial expression, increased autophagy, and decreased gluconeogenesis-associated transcriptional activity (23).

\section{Dormancy in C. neoformans studied in vitro}

In the previous study using the mouse model, the authors were able to generate as few as $10^{4}$ dormant yeast cells even after pooling several mouse lungs, which is obviously insufficient to study basic biological processes that would allow characterization of dormancy. Therefore, the authors worked on an in vitro model to enable them to generate a high number of dormant yeast cells. Recently, they released their study of the standardized conditions allowing the generation of yeast cells harboring a phenotype close to that of dormant cells generated in the lungs of infected mice (95). These conditions are based on a combination of low oxygen and 
limited nutrients inspired by the Wayne and the Loebel models, two well-documented models of conditions that allow generation of quiescent $M$. tuberculosis (104). After stationary phase in yeast peptone dextrose (YPD) and exposure to anaerobiosis and nutrient starvation during 8 days, the authors observed that $95 \%$ of yeast cells were viable, with few dead cells. They demonstrated that cells were not apoptotic upon TUNEL staining. Over time, these yeast cells showed a decreased culturability on YPD agar plates, ending with about $1 \%$ of the cells still able to grow on agar at day 8 of incubation. The phenotype observed in the in vivo subpopulation was resumed with delayed growth (increased latency) and low stress response (95). In total, the population obtained was homogeneously composed of cells characterized as viable but nonculturable cells (VBNCs) (Figure 1), a phenotype well known in many bacteria and first described in 1982 in E. coli (105). Among fungi, this phenotype has been described in S. cerevisiae (106), in Candida stellata (107), and in Brettanomyces bruxellensis grown in wine synthetic medium and induced by sulfur dioxide (108). C. neoformans VBNCs were induced by hypoxia and nutrient deprivation, and a proportion of them were able to be reactivated by vitamin $\mathrm{B}_{5}$ (pantothenic acid) with a doubling number of culturable cells (Figure 1). Notably, it has been shown in a specific model in E. coli that the VBNCs were potentially unable to reactivate (109). Pantothenic acid is known to play a role in the process of division (cell cycle) and in the quorum-sensing phenomenon (110). The use of diluted medium (which is poor in nutrients) to try to reactivate VBNCs was attempted, reflecting the observation that rich medium can be deleterious and induce death (111). Diluted medium did not lead to reactivation of more cells than rich medium, but rather, the cells that did reactivate exhibited faster growth and an increased doubling time in comparison with rich medium. This cell phenotype induced by diluted medium has been called rewiring (95). Finally, drawing on large omics methods, the authors of this study were able to show that C. neoformans VBNCs harbored a decreased and specific metabolic activity on the basis of phenotypic microarray, transcriptome, secretome, and proteome analyses (95). Specifically, the fatty acid pathway was required for the maintenance and the viability of the VBNCs, and quorum sensing and mTOR pathways seemed to play an important role in generating and/or maintaining the phenotype. Interestingly, acetyl-CoA is a key precursor for both fatty acids and pantothenic acid, suggesting that regulation of acetyl-CoA is a major factor for the generation of VBNCs (112). Based on these findings, a basic model of the evolution of C. neoformans yeast cells from logarithmic phase to dormancy and dead cells can be summarized as depicted in Figure 1.

An analysis of the bulk population of yeasts maintained 8 days in nutrient deprivation and anaerobiosis to generate VBNCs identified another subpopulation of yeasts that are still able and ready to grow on agar-rich medium. This population can be considered as persister cells. Persister cells have been described in a population of bacteria exposed to fungicidal antibiotics as the small proportion of bacteria able to tolerate spontaneously and stochastically lytic drugs via different mechanisms $(113,114)$. It has been shown that persisters and VBNCs can coexist in a specific model in the bacterium Vibrio vulnificus (115). Persister cells have been described in Candida albicans biofilm (116) and seem to play a role in recurrent infection in human oral candidiasis associated with natural biofilm (117). In VBNC-inducing conditions in C. neoformans, remaining cells able to grow rapidly after long exposure to harsh conditions could be related to such persister cells, since a particular metabolism seems to occur distinct from that of the VBNCs, which need a specific stimulus to grow again. This needs to be studied in detail in future research.

\section{Relevance and unsolved questions}

The recent study highlighting the capacity of $C$. neoformans to switch to VBNCs can be viewed as a model to explore dormancy and metabolism in this organism and in pathogenic fungi in general. Indeed, this phenotype per se has not yet been observed biologically in human infection, but experimental conditions and the number of yeast cells needed to obtain the demonstration are clearly not compatible with what can be recovered from the cerebrospinal fluid of a patient with CM. The observation of yeast cells in the cerebrospinal fluid of patients after 7 days of induction therapy with a negative culture on regular medium obviously raises the question of whether these yeasts are VBNCs. Being involved in clinical diagnosis, I have observed that the morphology of these nonculturable yeasts is abnormal and close to that observed in murine infection and called Drop Cn (see above) (8). Indeed, dead yeasts are known to persist and keep their intact shape, although different stainings can help differentiate them from regular and living yeasts (8). VBNCs, or at least part of the VBNC population, have proven to be reactivatable by pantothenic acid, part of the demonstration that these cells are VBNCs. The mechanism behind the specific reactivation has yet to be elucidated. Pantothenic acid (vitamin $\mathrm{B}_{5}$ ) is a precursor of coenzyme $\mathrm{A}$, an essential compound that participates in the metabolism of fatty acids, carbohydrates, and proteins through the formation of various active thioesters and promotes virulence and growth (118). Indeed, fatty acids have been shown to be critical in VBNCs (95). VBNCs obtained in C. neoformans can be considered as similar to those obtained from bacteria or parasites, as the definition relies on viability, culturability, and reactivation upon specific stimuli. Nevertheless, the conditions allowing the generation of VBNCs and the stimuli that reactivate the population are different in different organisms. Among organisms, many common conditions of induction rely on stresses including starvation, low oxygen, low temperature, desiccation, or a combination of these. On the other hand, resuscitation conditions are extremely variable depending on the organism, such as increased nutrient availability, temperature modifications, addition of chemicals, or addition of host factors (119).

The biology of dormancy in C. neoformans is a budding field, and yet there are many more questions than answers. We still lack data on the effect of antifungal drugs on dormant yeast cells, because an experimental setting that allows demonstration of the effect or absence of effect is not easy to implement in dormant cells that are intrinsically not cultivable. Indeed, turning on dormancy with some VBNC inductors that remain to be discovered would definitely aid in treating acute infection. On the other hand, inducing VBNCs could also cause relapse by producing insensitivity to current antifungal strategies. These factors need to be addressed. Moreover, we have no data yet on the possible extension of the VBNC phenotype to clinical isolates of C. neoformans type VNI and to other phylogenetic lineages or species. There is 
a chance that all clinical isolates could have varying propensities to generate VBNCs, and so the impact on infection could be variable, as already shown for phenotypes of interaction with macrophages $(8,83)$. We are currently exploring the effect of the host on the induction and maintenance of VBNCs with regard to the level of activation of primary monocytes. Another important subject we are currently exploring is the impact of VBNC metabolism on the physiology of macrophages. Both studies aim to understand the interplay between host and fungal metabolisms, opening the way to discovery of specific pathways that could be modulated to push the system in one or the other direction (more killing or less proliferation of yeasts).

\section{Conclusions}

In summary, C. neoformans can adapt fantastically to various environments, even very drastic ones, such as 8 days of complete anaerobiosis without extracellular nutrients. C. neoformans uses strategies to resist these conditions. It is first perfectly able to enter quiescence in nutrient starvation conditions (stationary phase) or to be pushed into dormancy under additional anaerobiosis exposure. In vivo, one can imagine that VBNCs/dormant yeasts are most likely hidden in the innate immune cells for years before being able to reactivate and multiply in the body of immunocompromised patients but also in the environment. This makes C. neoformans the first relevant pathogenic organism in which to study fungal dormancy and its role in pathogenesis in humans.

\section{Acknowledgments}

I thank Françoise Dromer for her helpful comments and support and Stéphane Bretagne and Arturo Casadevall for their continuous support. I thank Aude Sturny-Leclère, who worked hard on dormancy in C. neoformans for several years in my group. I thank Christine Schmitt and Olivier Gorgette from the electron microscopy facility (UTech UBI) who helped generate electron microscopy pictures. No funding sources supported this work. This article is dedicated to the memory of my father, for whom the question of "in between life and death" is not relevant anymore.

Address correspondence to: Alexandre Alanio, Molecular Mycology Unit, Institut Pasteur, 25-28 rue du Dr Roux, 75724 Paris CEDEX 15, France. Phone: 33.1406.13255; Email: alexandre. alanio@pasteur.fr.
1. Dromer F, Mathoulin-Pélissier S, Launay O, Lortholary O, French Cryptococcosis Study Group. Determinants of disease presentation and outcome during cryptococcosis: the CryptoA/D study. PLoS Med. 2007;4(2):e21.

2. Perfect JR, Lang SD, Durack DT. Chronic cryptococcal meningitis: a new experimental model in rabbits. Am J Pathol. 1980;101(1):177-194.

3. Casadevall A, Steenbergen JN, Nosanchuk JD. 'Ready made' virulence and 'dual use' virulence factors in pathogenic environmental fungi - the Cryptococcus neoformans paradigm. Curr Opin Microbiol. 2003;6(4):332-337.

4. Wozniak KL, Levitz SM. Cryptococcus neoformans enters the endolysosomal pathway of dendritic cells and is killed by lysosomal components. Infect Immun. 2008;76(10):4764-4771.

5. Murphy JW, Zhou A, Wong SC. Direct interactions of human natural killer cells with Cryptococcus neoformans inhibit granulocyte-macrophage colony-stimulating factor and tumor necrosis factor alpha production. Infect Immun. 1997;65(11):4564-4571.

6. Fries BC, Taborda CP, Serfass E, Casadevall A. Phenotypic switching of Cryptococcus neoformans occurs in vivo and influences the outcome of infection. JClin Invest. 2001;108(11):1639-1648.

7. Feldmesser M, Kress Y, Novikoff P, Casadevall A. Cryptococcus neoformans is a facultative intracellular pathogen in murine pulmonary infection. Infect Immun. 2000;68(7):4225-4237.

8. Alanio A, Desnos-Ollivier M, Dromer F. Dynamics of Cryptococcus neoformans-macrophage interactions reveal that fungal background influences outcome during cryptococcal meningoencephalitis in humans. mBio. 2011;2(4):e00158-11.

9. Alvarez M, Casadevall A. Cell-to-cell spread and massive vacuole formation after Cryptococcus neoformans infection of murine macrophages. BMC Immunol. 2007;8:16.

10. Alvarez M, Casadevall A. Phagosome extrusion and host-cell survival after Cryptococcus neoformans phagocytosis by macrophages. Curr Biol. 2006;16(21):2161-2165.

11. Johnston SA, May RC. The human fungal pathogen Cryptococcus neoformans escapes macrophages by a phagosome emptying mechanism that is inhibited by Arp2/3 complex-mediated actin polymerisation. PLoS Pathog. 2010;6(8):e1001041.

12. Dragotakes Q, Fu MS, Casadevall A. Dragotcytosis: elucidation of the mechanism for Cryptococcus neoformans macrophage-to-macrophage transfer. J Immunol. 2019;202(9):2661-2670.

13. Levitz SM, Nong SH, Seetoo KF, Harrison TS, Speizer RA, Simons ER. Cryptococcus neoformans resides in an acidic phagolysosome of human macrophages. Infect Immun. 1999;67(2):885-890.

14. Sorrell TC, et al. Cryptococcal transmigration across a model brain blood-barrier: evidence of the Trojan horse mechanism and differences between Cryptococcus neoformans var. grubii strain H99 and Cryptococcus gattii strain R265. Microbes Infect. 2016;18(1):57-67.

15. Charlier C, Nielsen K, Daou S, Brigitte M, Chretien F, Dromer F. Evidence of a role for monocytes in dissemination and brain invasion by Cryptococcus neoformans. Infect Immun. 2009;77(1):120-127.

16. Chang YC, Kwon-Chung KJ. Complementation of a capsule-deficient mutation of Cryptococcus neoformans restores its virulence. Mol Cell Biol. 1994;14(7):4912-4919.

17. $\mathrm{Ma} \mathrm{H}$, et al. The fatal fungal outbreak on Vancouver Island is characterized by enhanced intracellular parasitism driven by mitochondrial regulation. Proc Natl Acad Sci U S A. 2009;106(31):12980-12985.

18. Mansour MK, Vyas JM, Levitz SM. Dynamic virulence: real-time assessment of intracellular pathogenesis links Cryptococcus neofor- mans phenotype with clinical outcome. mBio. 2011;2(5):e00217-11.

19. Derengowski Lda S, et al. The transcriptional response of Cryptococcus neoformans to ingestion by Acanthamoeba castellanii and macrophages provides insights into the evolutionary adaptation to the mammalian host. Eukaryotic Cell. 2013;12(5):761-774.

20. Fan W, Kraus PR, Boily MJ, Heitman J. Cryptococcus neoformans gene expression during murine macrophage infection. Eukaryotic Cell. 2005;4(8):1420-1433.

21. Hu G, Cheng PY, Sham A, Perfect JR, Kronstad JW. Metabolic adaptation in Cryptococcus neoformans during early murine pulmonary infection. Mol Microbiol. 2008;69(6):1456-1475.

22. Steen BR, et al. Cryptococcus neoformans gene expression during experimental cryptococcal meningitis. Eukaryotic Cell. 2003;2(6):1336-1349.

23. Alanio A, Vernel-Pauillac F, Sturny-Leclère A, Dromer F. Cryptococcus neoformans host adaptation: toward biological evidence of dormancy. mBio. 2015;6(2):e02580-14.

24. Rajasingham R, et al. Global burden of disease of HIV-associated cryptococcal meningitis: an updated analysis. Lancet Infect Dis. 2017;17(8):873-881.

25. Bitar D, et al. Population-based analysis of invasive fungal infections, France, 2001-2010. Emerging Infect Dis. 2014;20(7):1149-1155.

26. Marr KA, et al. A multicenter, longitudinal cohort study of cryptococcosis in human immunodeficiency virus-negative people in the United States. Clin Infect Dis. 2020;70(2):252-261.

27. Rhodes J, et al. Tracing genetic exchange and biogeography of Cryptococcus neoformans var. grubii at the global population level. Genetics. 2017;207(1):327-346.

28. Desnos-Ollivier M, et al. Mixed infections and in vivo evolution in the human fungal pathogen Cryptococcus neoformans. mBio. 2010;1(1):e00091-10. 
29. Xu J, Mitchell TG. Comparative gene genealogical analyses of strains of serotype AD identify recombination in populations of serotypes $\mathrm{A}$ and $D$ in the human pathogenic yeast Cryptococcus neoformans. Microbiology (Reading, Engl). 2003;149(pt 8):2147-2154.

30. Xu J, Vilgalys R, Mitchell TG. Multiple gene genealogies reveal recent dispersion and hybridization in the human pathogenic fungus Cryptococcus neoformans. Mol Ecol. 2000;9(10):1471-1481.

31. Bovers $\mathrm{M}$, et al. Unique hybrids between the fungal pathogens Cryptococcus neoformans and Cryptococcus gattii. FEMS Yeast Res. 2006;6(4):599-607.

32. Emmons CW. Isolation of Cryptococcus neoformans from soil. J Bacteriol. 1951;62(6):685-690.

33. Fink JN, Barboriak JJ, Kaufman L. Cryptococcal antibodies in pigeon breeders' disease. JAllergy. 1968;41(5):297-301.

34. Passoni LF, Wanke B, Nishikawa MM, Lazéra MS. Cryptococcus neoformans isolated from human dwellings in Rio de Janeiro, Brazil: an analysis of the domestic environment of AIDS patients with and without cryptococcosis. Med Mycol. 1998;36(5):305-311.

35. Brito-Santos F, et al. Environmental isolation of Cryptococcus gattii VGII from indoor dust from typical wooden houses in the deep Amazonas of the Rio Negro basin. PLoS One. 2015;10(2):e0115866.

36. Nosanchuk JD, Shoham S, Fries BC, Shapiro DS, Levitz SM, Casadevall A. Evidence of zoonotic transmission of Cryptococcus neoformans from a pet cockatoo to an immunocompromised patient. Ann Intern Med. 2000;132(3):205-208.

37. Fessel WJ. Cryptococcal meningitis after unusual exposures to birds. $N$ Engl JMed. 1993;328(18):1354-1355.

38. Shrestha RK, Stoller JK, Honari G, Procop GW, Gordon SM. Pneumonia due to Cryptococcus neoformans in a patient receiving infliximab: possible zoonotic transmission from a pet cockatiel. Respir Care. 2004;49(6):606-608.

39. Fraison JB, et al. Pulmonary cryptococcosis in a patient with Crohn's disease treated with prednisone, azathioprine and adalimumab: exposure to chicken manure as a source of contamination. JCrohns Colitis. 2013;7(1):e11-e14.

40. Staib F, Schulz-Dieterich J. Cryptococcus neoformans in fecal matter of birds kept in cages - control of Cr. neoformans habitats. Zentralbl Bakteriol Mikrobiol Hyg B. 1984;179(2):179-186.

41. Wang CY, Wu HD, Hsueh PR. Nosocomial transmission of cryptococcosis. N Engl J Med. 2005;352(12):1271-1272.

42. Ingram CW, Haywood HB, Morris VM, Allen RL, Perfect JR. Cryptococcal ventricular-peritoneal shunt infection: clinical and epidemiological evaluation of two closely associated cases. Infect Control Hosp Epidemiol. 1993;14(12):719-722.

43. Kanj SS, et al. Fungal infections in lung and heartlung transplant recipients. Report of 9 cases and review of the literature. Medicine (Baltimore). 1996;75(3):142-156.

44. Ooi BS, Chen BT, Lim CH, Khoo OT, Chan DT. Survival of a patient transplanted with a kidney infected with Cryptococcus neoformans. Transplantation. 1971;11(4):428-429.
45. Baddley JW, et al. Transmission of Cryptococcus neoformans by organ transplantation. Clin Infect Dis. 2011;52(4):e94-e98.

46. Ye F, Xie JX, Zeng QS, Chen GQ, Zhong SQ, Zhong NS. Retrospective analysis of $76 \mathrm{immu}-$ nocompetent patients with primary pulmonary cryptococcosis. Lung. 2012;190(3):339-346.

47. Zeng Y, et al. Clinicopathologic and ultrastructural study of non-HIV-related primary pulmonary cryptococcosis in China: report of 43 cases. Ultrastruct Pathol. 2011;35(1):19-25.

48. Setianingrum F, Rautemaa-Richardson R, Denning DW. Pulmonary cryptococcosis: a review of pathobiology and clinical aspects. Med Mycol. 2019;57(2):133-150.

49. Christianson JC, Engber W, Andes D. Primary cutaneous cryptococcosis in immunocompetent and immunocompromised hosts. Med Mycol. 2003;41(3):177-188

50. Neuville S, et al. Primary cutaneous cryptococcosis: a distinct clinical entity. Clin Infect Dis. 2003;36(3):337-347.

51. Giles SS, Dagenais TR, Botts MR, Keller NP, Hull $\mathrm{CM}$. Elucidating the pathogenesis of spores from the human fungal pathogen Cryptococcus neoformans. Infect Immun. 2009;77(8):3491-3500.

52. Goldman DL, et al. Serologic evidence for Cryptococcus neoformans infection in early childhood. Pediatrics. 2001;107(5):E66.

53. Davis J, et al. Serologic evidence for regional differences in pediatric cryptococcal infection. Pediatr Infect Dis J. 2007;26(6):549-551.

54. Park BJ, Wannemuehler KA, Marston BJ, Govender N, Pappas PG, Chiller TM. Estimation of the current global burden of cryptococcal meningitis among persons living with HIV/AIDS. AIDS. 2009;23(4):525-530.

55. Hurtado JC, et al. Mortality due to Cryptococcus neoformans and Cryptococcus gattii in low-income settings: an autopsy study. Sci Rep. 2019;9(1):7493.

56. Galanis E, Macdougall L, Kidd S, Morshed M, British Columbia Cryptococcus gattii Working Group. Epidemiology of Cryptococcus gattii, British Columbia, Canada, 1999-2007. Emerging Infect Dis. 2010;16(2):251-257.

57. Atkinson AJ, Bennett JE. Experience with a new skin test antigen prepared from Cryptococcus neoformans. Am Rev Respir Dis. 1968;97(4):637-643.

58. Baker RD. The primary pulmonary lymph node complex of crytptococcosis. Am J Clin Pathol. 1976;65(1):83-92.

59. Bao F, Tan H, Liu W, Li Y, Li H. Pediatric cryptococcal lymphadenitis in the absence of AIDS: case report and literature review. Case Rep Pediatr. 2013;2013:563081.

60. Gurung J, Lyngdoh WV, Khyriem AB. Isolated cervical cryptococcal lymphadenitis without meningitis in an immunocompetent human immunodeficiency virus-negative child: a rare case report [Published online September 1, 2014]. JMM Case Rep. https://doi.org/10.1099/jmmcr.0.002592.

61. Jha DK, Jha AK, Singh RK. An atypical initial presentation of AIDS as cryptococcal lymphadenitis. OxfMed Case Reports. 2018;2018(11):omy083.

62. Sood A, Chandel LR, Chauhan S, Thakur K, Jaryal SC. A rare case of primary supraclavicular lymphadenitis due to cryptococcus neoformans in an HIV infected patient. J Clin Diagn Res. 2014;8(2):137-138.

63. Dogbey P, Golden M, Ngo N. Cryptococcal lymphadenitis: an unusual initial presentation of HIV infection. BMJ Case Rep. 2013;2013:bcr2013010316.

64. Kawamoto K, et al. Clinicopathological features of cryptococcal lymphadenitis and a review of literature. JClin Exp Hematop. 2017;57(1):26-30.

65. Putignani L, et al. Cryptococcal lymphadenitis as a manifestation of immune reconstitution inflammatory syndrome in an HIV-positive patient: a case report and review of the literature. Int $J$ Immunopathol Pharmacol. 2008;21(3):751-756.

66. Jagadha V, Andavolu RH, Huang CT. Granulomatous inflammation in the acquired immune deficiency syndrome. Am JClin Pathol. 1985;84(5):598-602.

67. Hill JO. $\mathrm{CD}^{+}{ }^{+} \mathrm{T}$ cells cause multinucleated giant cells to form around Cryptococcus neoformans and confine the yeast within the primary site of infection in the respiratory tract. JExp Med. 1992;175(6):1685-1695.

68. Garcia-Hermoso D, Janbon G, Dromer F. Epidemiological evidence for dormant Cryptococcus neoformans infection. JClin Microbiol. 1999;37(10):3204-3209.

69. Saha DC, et al. Serologic evidence for reactivation of cryptococcosis in solid-organ transplant recipients. Clin Vaccine Immunol. 2007;14(12):1550-1554.

70. Dromer F, Ronin O, Dupont B. Isolation of Cryptococcus neoformans var. gattii from an Asian patient in France: evidence for dormant infection in healthy subjects. JMed Vet Mycol. 1992;30(5):395-397.

71. Liechty CA, et al. Asymptomatic serum cryptococcal antigenemia and early mortality during antiretroviral therapy in rural Uganda. Trop Med Int Health. 2007;12(8):929-935.

72. Andama AO, et al. Prevalence and outcomes of cryptococcal antigenemia in HIV-seropositive patients hospitalized for suspected tuberculosis in Uganda. J Acquir Immune Defic Syndr. 2013;63(2):189-194.

73. Micol R, et al. Prevalence, determinants of positivity, and clinical utility of cryptococcal antigenemia in Cambodian HIV-infected patients. J Acquir Immune Defic Syndr. 2007;45(5):555-559.

74. Temfack E, et al. Cryptococcal antigen screening in asymptomatic HIV-infected antiretroviral naive patients in Cameroon and evaluation of the new semi-quantitative Biosynex CryptoPS test. Front Microbiol. 2018;9:409.

75. Chammard TB, Temfack E, Lortholary O, Alanio A. Diagnostic and therapeutic strategies in cryptococcosis: impact on outcome. Mem Inst Oswaldo Cruz. 2018;113(7):e180050.

76. Shi $\mathrm{M}$, et al. Real-time imaging of trapping and urease-dependent transmigration of Cryptococcus neoformans in mouse brain. JClin Invest. 2010;120(5):1683-1693.

77. Shi M, Colarusso P, Calaruso P, Mody CH. Real-time in vivo imaging of fungal migration to the central nervous system. Cell Microbiol. 2012;14(12):1819-1827.

78. French N, et al. Cryptococcal infection in a cohort of HIV-1-infected Ugandan adults. AIDS. 
2002;16(7):1031-1038.

79. Perfect JR, et al. Clinical practice guidelines for the management of cryptococcal disease: 2010 update by the infectious diseases society of america. Clin Infect Dis. 2010;50(3):291-322.

80. Molloy SF, et al. Antifungal combinations for treatment of Cryptococcal Meningitis in Africa. N Engl JMed. 2018;378(11):1004-1017.

81. Day JN, et al. Combination antifungal therapy for cryptococcal meningitis. New Engl J Med. 2013;368(14):1291-1302.

82. Ou XT, et al. Genotypes coding for mannose-binding lectin deficiency correlated with cryptococcal meningitis in HIV-uninfected Chinese patients. J Infect Dis. 2011;203(11):1686-1691.

83. Sabiiti W, et al. Efficient phagocytosis and laccase activity affect the outcome of HIV-associated cryptococcosis. JClin Invest. 2014;124(5):2000-2008.

84. Gray JV, Petsko GA, Johnston GC, Ringe D, Singer RA, Werner-Washburne M. "Sleeping beauty": quiescence in Saccharomyces cerevisiae. Microbiol Mol Biol Rev. 2004;68(2):187-206.

85. Rittershaus ES, Baek SH, Sassetti CM. The normalcy of dormancy: common themes in microbial quiescence. Cell Host Microbe. 2013;13(6):643-651.

86. Finkel SE. Long-term survival during stationary phase: evolution and the GASP phenotype. Nat Rev Microbiol. 2006;4(2):113-120.

87. Corper HJ, Cohn ML. The viability and virulence of old cultures of tubercle bacilli; studies on 30 -year-old broth cultures maintained at 37 degrees C. Tubercle. 1951;32(11):232-237.

88. Gengenbacher M, Rao SP, Pethe K, Dick T. Nutrient-starved, non-replicating Mycobacterium tuberculosis requires respiration, ATP synthase and isocitrate lyase for maintenance of ATP homeostasis and viability. Microbiology (Reading, Engl). 2010;156(pt 1):81-87.

89. Setlow P. Germination of spores of Bacillus species: what we know and do not know. J Bacteriol. 2014;196(7):1297-1305.

90. Lillie SH, Pringle JR. Reserve carbohydrate metabolism in Saccharomyces cerevisiae: responses to nutrient limitation. J Bacteriol. 1980;143(3):1384-1394.

91. Jamieson DJ. Oxidative stress responses of the yeast Saccharomyces cerevisiae. Yeast. 1998;14(16):1511-1527.

92. Davidson GS, et al. The proteomics of quiescent and nonquiescent cell differentiation in yeast stationary-phase cultures. Mol Biol Cell. 2011;22(7):988-998.
93. Takeo K, Tanaka R, Miyaji M, Nishimura K. Unbudded G2 as well as G1 arrest in the stationary phase of the basidiomycetous yeast Cryptococcus neoformans. FEMS Microbiol Lett. 1995;129(2-3):231-235.

94. Mochizuki T, Tanaka S, Saito Y, Watanabe S. Mitochondrial kinetics during mitosis in Cryptococcus neoformans - an ultrastructural study. Mycoses. 1989;32(1):7-13.

95. Hommel B, et al. Cryptococcus neoformans resists to drastic conditions by switching to viable but non-culturable cell phenotype. PLoS Pathog. 2019;15(7):e1007945

96. Diamond RD, Root RK, Bennett JE. Factors influencing killing of Cryptococcus neoformans by human leukocytes in vitro. J Infect Dis. 1972;125(4):367-376.

97. Green L, Petersen B, Steimel L, Haeber P, Current W. Rapid determination of antifungal activity by flow cytometry. J Clin Microbiol. 1994;32(4):1088-1091.

98. Nicola AM, Robertson EJ, Albuquerque P, Derengowski Lda S, Casadevall A. Nonlytic exocytosis of Cryptococcus neoformans from macrophages occurs in vivo and is influenced by phagosomal pH. mBio. 2011;2(4):e00167-11.

99. Hardwick JM. Do fungi undergo apoptosislike programmed cell death? mBio 2018;9(4):e00948-18.

100.Semighini CP, Averette AF, Perfect JR, Heitman J. Deletion of Cryptococcus neoformans AIF ortholog promotes chromosome aneuploidy and fluconazole-resistance in a metacaspase-independent manner. PLoS Pathog. 2011;7(11):e1002364.

101.Aouacheria A, et al. Comment on "Sterilizing immunity in the lung relies on targeting fungal apoptosis-like programmed cell death". Science. 2018;360(6395):eaar6910.

102. Goulart L, et al. Cryptococcus neoformans and Cryptococcus gattii genes preferentially expressed during rat macrophage infection. $\mathrm{Med}$ Mycol.2010;48(7):932-941.

103. Chen Y, et al. The Cryptococcus neoformans transcriptome at the site of human meningitis. mBio. 2014;5(1):e01087-e01013.

104.Gengenbacher M, Kaufmann SH. Mycobacterium tuberculosis: success through dormancy. FEMS Microbiol Rev. 2012;36(3):514-532.

105. Xu HS, Roberts N, Singleton FL, Attwell RW, Grimes DJ, Colwell RR. Survival and viability of nonculturable Escherichia coli andVibrio cholerae in the estuarine and marine environment. Microb Ecol. 1982;8(4):313-323.
106.Salma M, Rousseaux S, Sequeira-Le Grand A, Divol B, Alexandre H. Characterization of the viable but nonculturable (VBNC) state in Saccharomyces cerevisiae. PLoS One. 2013;8(10):e77600

107. Mills DA, Johannsen EA, Cocolin L. Yeast diversity and persistence in botrytis-affected wine fermentations. Appl Environ Microbiol. 2002;68(10):4884-4893.

108. du Toit WJ, Pretorius IS, Lonvaud-Funel A. The effect of sulphur dioxide and oxygen on the viability and culturability of a strain of Acetobacter pasteurianus and a strain of Brettanomyces bruxellensis isolated from wine. J Appl Microbiol. 2005;98(4):862-871.

109. Liew KL, Jee JM, Yap I, Yong PV. In vitro analysis of metabolites secreted during infection of lung epithelial cells by Cryptococcus neoformans. PLoS One. 2016;11(4):e0153356.

110.Albuquerque $\mathrm{P}$, et al. Quorum sensing-mediated, cell density-dependent regulation of growth and virulence in Cryptococcus neoformans. mBio. 2013;5(1):e00986-e00913.

111. Nofal M, Zhang K, Han S, Rabinowitz JD. mTOR inhibition restores amino acid balance in cells dependent on catabolism of extracellular protein. Mol Cell. 2017;67(6):936-946.e5.

112. Martinez DL, Tsuchiya Y, Gout I. Coenzyme A biosynthetic machinery in mammalian cells. Biochem Soc Trans. 2014;42(4):1112-1117.

113. Lewis K. Persister cells, dormancy and infectious disease. Nat Rev Microbiol. 2007;5(1):48-56.

114. Kim JS, Wood TK. Persistent persister misperceptions. Front Microbiol. 2016;7:2134.

115. Ayrapetyan M, Williams TC, Baxter R, Oliver JD. Viable but nonculturable and persister cells coexist stochastically and are induced by human serum. Infect Immun. 2015;83(11):4194-4203.

116. LaFleur MD, Kumamoto CA, Lewis K. Candida albicans biofilms produce antifungal-tolerant persister cells. Antimicrob Agents Chemother. 2006;50(11):3839-3846.

117. Lafleur MD, Qi Q, Lewis K. Patients with longterm oral carriage harbor high-persister mutants of Candida albicans. Antimicrob Agents Chemother. 2010;54(1):39-44.

118. Spry C, Kirk K, Saliba KJ. Coenzyme A biosynthesis: an antimicrobial drug target. FEMS Microbio Rev. 2008;32(1):56-106.

119. Zhao X, Zhong J, Wei C, Lin CW, Ding T. Current perspectives on viable but non-culturable state in foodborne pathogens. Front Microbiol. 2017;8:580. 